\title{
The abscopal effect in head-and-neck squamous cell carcinoma treated with radiotherapy and nivolumab: a case report and literature review
}

D. Forner $M D, *$ P. Horwich $M D_{,}^{\dagger}$ J.R. Trites $M D_{,}^{*}$ H. Hollenhorst $M D_{,}^{\ddagger}$ M. Bullock $M D, \$$ and N.W.D. Lamond MDI

\begin{abstract}
Introduction The abscopal effect is a rarely observed outcome of radiotherapy wherein there is a reduction in metastatic disease burden outside of the targeted treatment area. Likely due to an in situ vaccine effect of radiotherapy, the abscopal effect may be augmented by immunotherapy. This report is the first case of the abscopal effect observed in metastatic head-and-neck squamous cell carcinoma (HNSCC) treated with concurrent radiotherapy and single-agent nivolumab.
\end{abstract}

Case Description An otherwise healthy 57-year-old man underwent craniofacial resection and adjuvant chemoradiotherapy for advanced sinonasal squamous cell carcinoma. Distant metastatic disease developed shortly after primary treatment, and immunotherapy in the form of nivolumab was initiated. Subsequent oligometastatic progression despite immunotherapy prompted palliative radiotherapy to a single metastasis due to pending symptomatology. Post-radiotherapy, the abscopal effect was observed with all distant sites of metastatic disease shrinking. Five months following treatment, a sustained reduction in disease burden has been demonstrated.

Summary We present the first case of the abscopal effect in a patient with metastatic HNSCC treated with palliative radiotherapy concurrent with single-agent nivolumab immunotherapy, and only the third case of the abscopal effect in metastatic head-and-neck cancer. Dual treatment with immunotherapy and radiotherapy may be an important treatment option in the future, mediated through the abscopal effect.

Key Words Head-and -neck neoplasms, antineoplastics, radiation oncology, medical oncology

Curr Oncol. 2020 December:27(6)330-335

www.current-oncology.com

\section{INTRODUCTION}

Head-and-neck squamous cell carcinoma (HNSCC) is the 6th most common cancer worldwide, with over 500,000 new cases diagnosed each year ${ }^{1}$. Sinonasal cancer, constituting $5 \%$ of all head and neck malignancies, is a rare, aggressive type of head-and-neck cancer encompassing the nasal cavity and paranasal sinuses ${ }^{2,3}$.

Distant metastatic disease is rare for sinonasal cancer, occurring in approximately $5 \%$ of patients ${ }^{4}$. Treatment options remain limited in this setting, and there is an exceptionally high morbidity and mortality rate. Recently, immunotherapy has been used successfully in the management of treatment-resistant metastatic HNSCC, showing improved survival compared with conventional systemic therapy ${ }^{5-7}$. Ongoing trials are investigating the role of first-line immunotherapy alone or in combination with chemotherapy in treatment-naïve metastatic disease ${ }^{8}$.

Radiotherapy is capable of generating the abscopal effect, whereby there is a reduction in tumour burden outside the targeted radiotherapy field. While occurrence of the abscopal effect is infrequent and unpredictable, the use 
of immunotherapy is believed to increase the likelihood of this occurrence ${ }^{9}$. There have been approximately 50 cases of abscopal effect reported in the literature, of which only 2 cases are of metastatic HNSCC ${ }^{10,11}$.

This report therefore presents the 3rd such case of a reduction in distant metastatic disease burden following locoregional radiotherapy and immunotherapy in a patient with HNSCC, and the first with single-agent nivolumab. We also highlight previous cases through systematic review, as well as the proposed mechanism of the abscopal effect.

\section{METHODS}

A single-reviewer (DF) systematic literature review was carried out using MEDLINE and Google Scholar. In brief, search terms included "abscopal," "neoplasms," and key words descriptive of head-and-neck cancer sites, for example "oropharynx." Google Scholar was searched with the terms "abscopal," "head and neck," and "carcinoma." Searches were performed from database inception to 25 December 2019. Snowballing and reference review techniques of relevant articles were performed in order to identify additional case reports that could potentially meet inclusion.

The MEDLINE search yielded twenty-three articles, of which none presented case reports or series of the abscopal effect in HNSCC. Google Scholar identified a single case report, and an additional case report was found upon reference searches of relevant abscopal effect reviews.

This manuscript was prepared in accordance with the CARE guidelines for the reporting of case reports ${ }^{12}$. Individual informed consent was granted by the patient for dissemination of case details.

\section{CASE DESCRIPTION}

The patient was an otherwise healthy 57-year-old man with past medical history significant for hypertension. He was a lifetime nonsmoker, and he drank alcohol occasionally. The patient presented with acute left upper eyelid ptosis and 3-week history of facial pain, mild nasal discharge, and congestion. Initial computed tomography (СT) imaging showed opacification of the left frontal sinus and ethmoid sinuses. There was destruction of the left superomedial orbital wall, with projection of a lobulated soft-tissue mass into the orbit causing displacement of the globe inferomedially. There was erosion of the posterior table of the left frontal sinus and lamina papyracea, without intracranial involvement (Figure 1).

Biopsies obtained during endonasal endoscopic surgery at this time revealed poorly differentiated nonkeratinizing squamous cell carcinoma (SCC). The patient was reviewed at the multidisciplinary tumour board, and primary surgical treatment of the tumour with adjuvant therapy was thought to offer the highest probability of oncologic control. Subsequently, the patient underwent craniofacial resection with en bloc removal of the left superomedial orbit, frontal and ethmoid sinuses, medial maxilla, and cribriform plate. As there was no evidence of regional nodal involvement on CT imaging or integrated positron emission tomography-CT (PET-CT), lymphadenectomy was not pursued. Surgical margins were negative
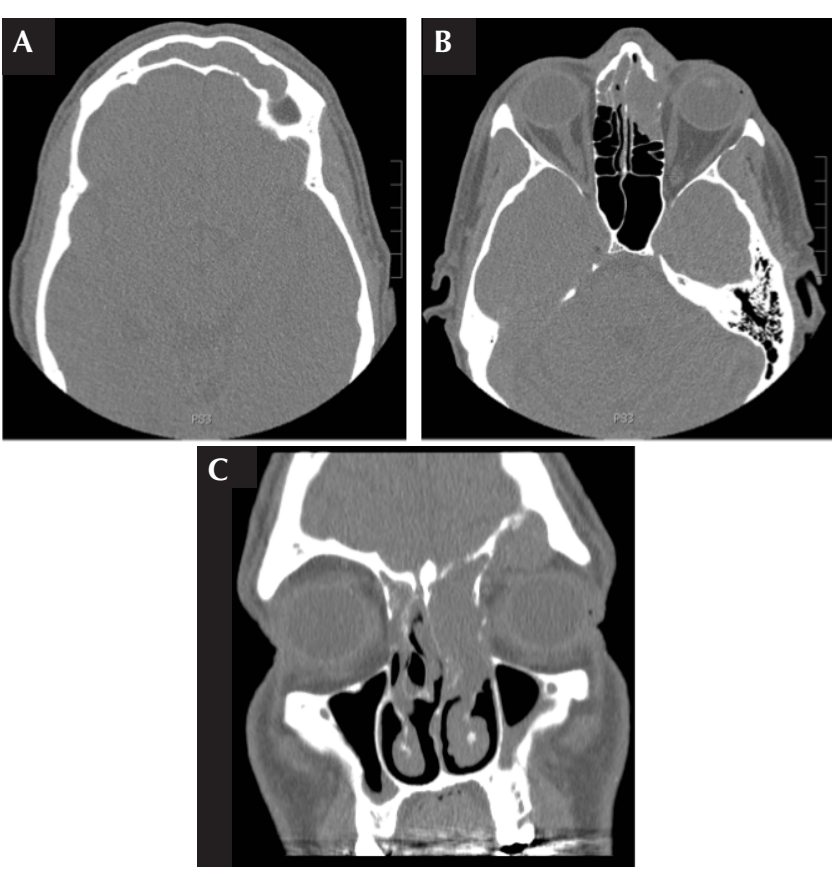

FIGURE 1 Patient's computed tomography imaging on initial presentation. (A) Axial image demonstrating opacification of the frontal sinus, with erosion and partial bone destruction involving the anterior and posterior tables of the left frontal sinus, creating microperforations to the anterior cranial fossa. (B) Axial image demonstrating erosion of the left lamina papyracea. (C) Coronal image demonstrating inferomedial displacement of the left globe from tumour mass extension into the orbit.

following the procedure, and the tumour was staged as pT4a. However, a close margin necessitated concurrent adjuvant chemoradiotherapy with 66 Gy over 33 fractions to the local site alongside 3 cycles of high-dose cisplatin administered as $100 \mathrm{mg} / \mathrm{m}^{2}$ intravenously every 21 days concurrent with radiotherapy.

Follow-up PET-CT imaging 4 months following primary treatment revealed a new fluorodeoxyglucose-avid, solitary $9 \mathrm{~mm}$ pulmonary nodule in the right lower lobe [Figure 2(A)]. Thoracic surgery consultation was sought, and the patient underwent an open right lower lobe segmentectomy and subcarinal nodal dissection for diagnostic and therapeutic purposes. The pathology was consistent with metastatic SCC, with pleural lymphatic invasion, and negative margins.

Given distant metastatic progression less than 6 months after completion of treatment, the patient was diagnosed with platinum-refractory metastatic HNSCC. Restaging CT imaging demonstrated 2 newly enlarged left cervical lymph nodes [Figure 2(B)], 2 additional new pulmonary nodules, and new pleural nodularity in keeping with further metastatic disease. Based on this, he began systematic therapy with nivolumab, $480 \mathrm{mg}$ fixed-dose treatment infused intravenously every 4 weeks.

The patient tolerated immunotherapy well, his only notable toxicity being initial hyperthyroidism progressing rapidly to hypothyroidism requiring levothyroxine replacement. The initial response to nivolumab was a prolonged period of asymptomatic and stable disease in 
the known multifocal sites of metastatic disease, until oligoprogression was observed after more than 1 year of treatment. At that time, CT demonstrated worsening left-sided pleural plaques with a new associated effusion, a newly enlarged $2 \mathrm{~cm}$ subcarinal lymph node, and an extraconal intraorbital lesion suggestive of metastasis (Figure 3).

The intraorbital mass was determined to be visionthreatening, and stereotactic radiotherapy was planned and administered urgently to prevent impending
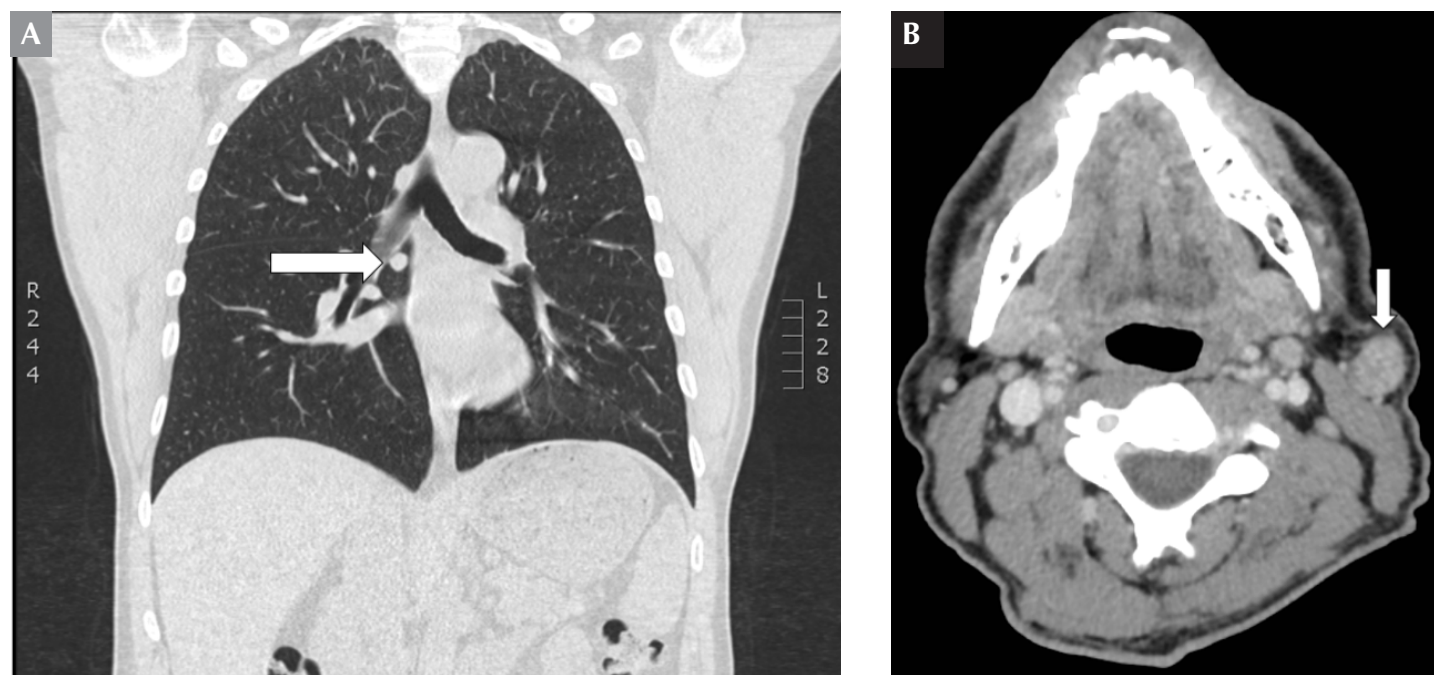

FIGURE 2 Computed tomography evidence of recurrent disease. (A) Coronal image demonstrating a new right pulmonary nodule measuring 9 mm (arrow). (B) Axial image demonstrating a $1.4 \mathrm{~cm}$ lesion inferior to the left parotid gland (arrow).
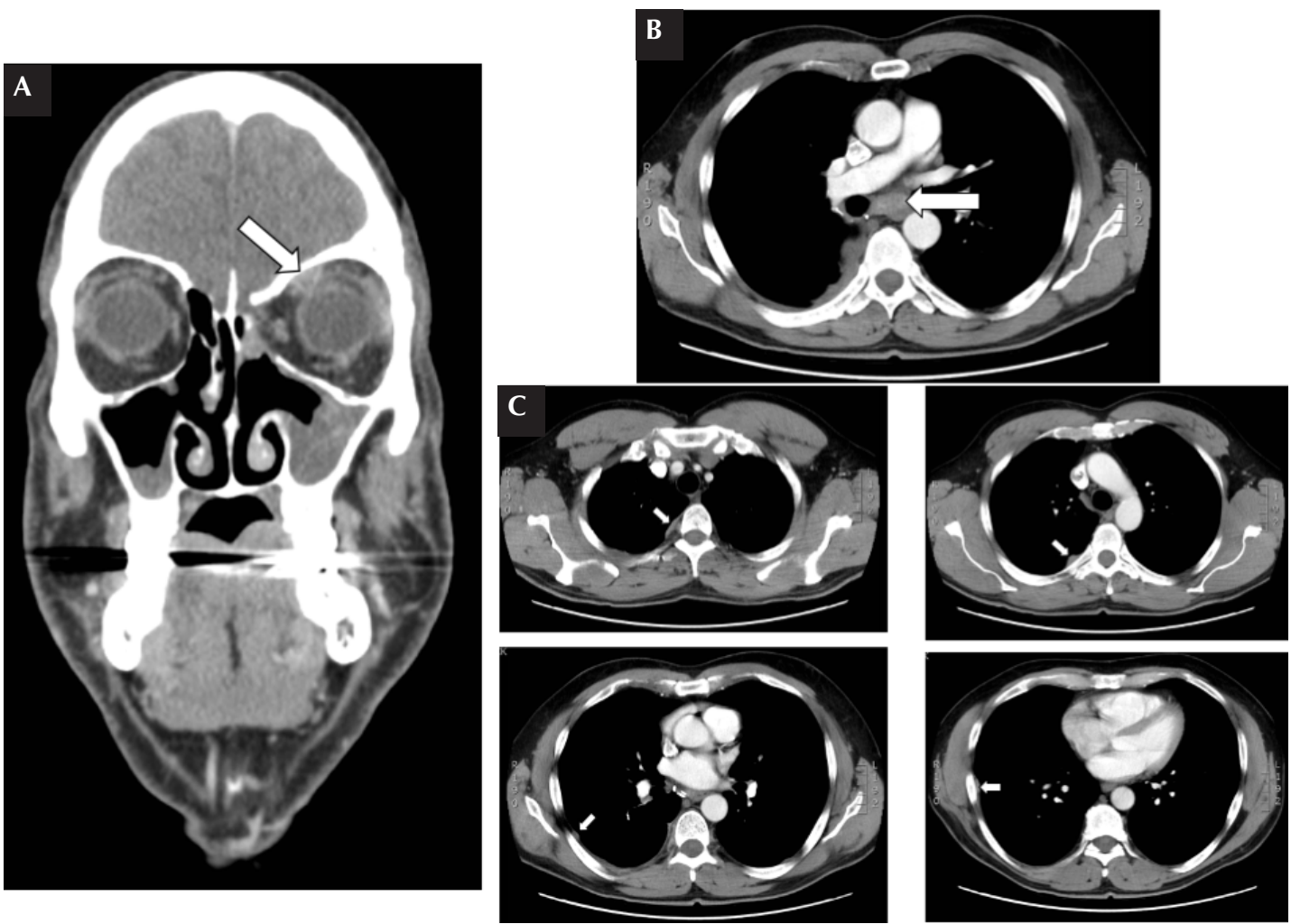

FIGURE 3 Computed tomography images demonstrating oligometastatic progression. (A) Coronal image of a new intraorbital lesion measuring $0.9 \times 1.0 \times 0.7 \mathrm{~cm}$, adjacent to the anterior aspect of the superior oblique muscles (arrow). (B) Axial image of a new enlarged subcarinal node, measuring $1.2 \times 2.8 \mathrm{~cm}$ (arrow). (C) Four axial images demonstrating pleural plaques (arrows). 
symptoms. Using a stereotactic radiation therapy protocol, 30 Gy in 5 fractions was prescribed to the $90 \%$ isodose line (Figure 4). The maximum dose within the target volume was $101.7 \%$ to the planning target volume, accounting for a $2 \mathrm{~mm}$ expansion from the clinical target volume. Treatment was tolerated well by the patient, despite the sum of total radiation doses exceeding normal tissue tolerance. Nivolumab monotherapy was continued during the time that radiotherapy was planned and administered.

Repeat imaging 1 month following radiotherapy showed significant tumour response of the intraorbital mass. Interestingly, non-irradiated metastatic disease within the thorax also showed significant improvement, including complete resolution of pleural nodularity and associated pleural effusion, resolution of multiple parenchymal nodules, and resolution of subcarinal lymphadenopathy. This response was felt to be in keeping with an abscopal effect, and nivolumab monotherapy was continued. Follow-up imaging 5 months later again showed stable disease, with no evidence of progression at locoregional or distant sites, and no major radiotherapy or immunotherapy toxicities.

\section{DISCUSSION}

This is the 3rd reported case of the abscopal effect in metastatic HNSCC. In this case, a patient with sinonasal sCC had metastatic recurrence despite primary combined-modality treatment and showed oligometastatic progression while on nivolumab immunotherapy. With the addition of palliative radiotherapy to prevent impending symptoms at a single site of progression, the ensuing abscopal effect generated a sustained response in metastatic disease outside the radiotherapy field.

Radiotherapy is known to have a dual effect on the immune system. Traditionally, radiotherapy was thought to be immunosuppressive, as demonstrated by the development of lymphopenia, the downregulation of antigen presenting cell (APC) co-stimulatory molecules, the release of transforming growth factor beta, and the increased infiltration of regulatory immune cells ${ }^{13-17}$. However, more recent evidence suggests that radiotherapy may in fact have immunogenic effects and be capable of immune system activation $^{18}$

Radiotherapy is capable of altering the tumour microenvironment. Through radiation-induced release of cytokines, infiltration of leucocytes, and alteration of tumour cell susceptibility, radiotherapy is capable of enhancing the immune system's ability to combat malignant cells $^{18}$. Radiotherapy induces type I interferon, allowing for activation of dendritic cells and T cells. In turn, type II interferon $(\gamma)$ is released, causing upregulation of major histocompatibility complex I (MHC I) and an increased presentation of tumour antigens to APCs. The cytokines and chemokines released through radiotherapy also result in a pro-inflammatory state and increased leucocyte infiltration including cytotoxic CD8+ T cells, natural killer cells, and macrophages. Alongside this infiltration, radiotherapy is capable of activating DCs and facilitating their migration to draining lymph nodes. Lastly, radiotherapy increases tumour cell susceptibility to immune system destruction through increased MHC I and Fas expression. Increased presentation of tumour antigens to APCs via MHC I allows for more robust $\mathrm{T}$ cell activation and related tumour death, and Fas is essential for T cell-mediated death. For a full review of radiotherapy and the immune system's interplay, see the review by Liu and colleagues ${ }^{18}$.

It is through the alteration of the immune system that radiotherapy is thought to exert its abscopal effects. The abscopal effect, from the root words $a b$ and scopos meaning "position away from" and "target," is the radiotherapyinduced reduction of tumour burden outside the radiotherapy target ${ }^{19}$. First described by Mole and colleagues in 1953, the abscopal effect has been variably described throughout the literature ${ }^{20}$. Through the radiotherapy-mediated

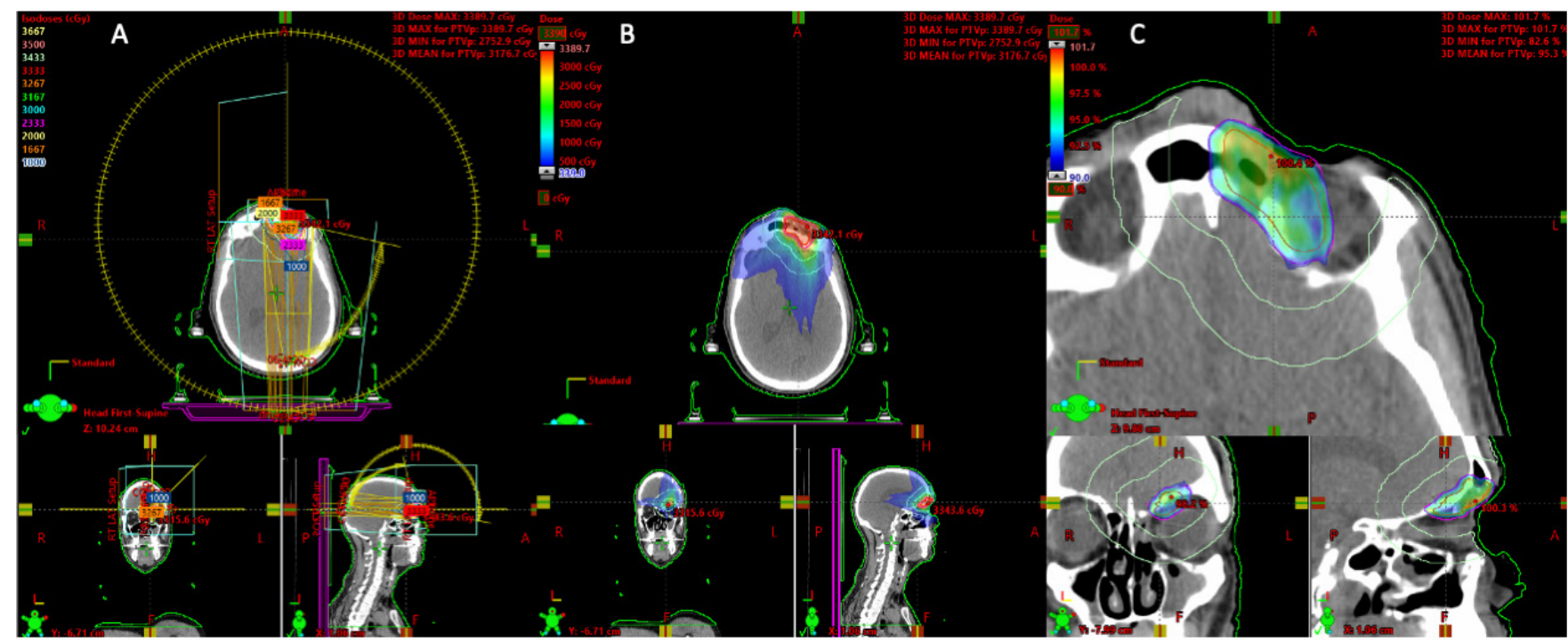

FIGURE 4 Palliative radiotherapy treatment plan. (A) Volumetric arc therapy plan showing Rapid Arcs arrangement and isodose lines. (B) Full dose distribution with colour wash. (C) Zoomed plan highlighting 90\% isodose line and planning treatment volume. Prescribed dose of 30 Gy in 5 fractions to $90 \%$ isodose line. 
destruction of tumour cells already described, the ensuing release of tumour neoantigens into the tumour microenvironment acts as an in situ vaccine. Activated T cells home to tumour cells throughout the body-both where the radiotherapy was targeted and to distant metastatic disease ${ }^{18}$.

Likely due to both the dual effect of radiotherapy on the immune system and the ability of cancer to evade immune detection, the occurrence of the abscopal effect is rare. Only 46 cases were described between 1969 and 2014, with the majority being primary tumours outside the head and neck ${ }^{21}$. However, with the recent development of immunotherapeutic agents such as nivolumab that reduce the inhibitory effects on the immune system, it has been proposed that the annual number of observed abscopal effects will increase ${ }^{18,22}$.

The addition of immunotherapy theoretically facilitates the mechanism of the abscopal effect, helping overcome the dual effect of radiotherapy and cancer immune evasion. It is believed that the sequence of treatment may play an important role, with immunotherapy preferentially leading the way ${ }^{23}$. The intensity of radiotherapy may also play a role, with stronger radiotherapy doses resulting in an increased likelihood of observing an abscopal effect. In the presented case, 30 Gy in 5 fractions was delivered, equating to 6 Gy per fraction, potentially contributing to a greater chance of producing an abscopal effect ${ }^{24}$. Finally, although necessary for primary treatment of many forms of head-and-neck cancer, surgical resection of the primary tumour and regional lymph nodes might reduce neoantigen availability, thereby decreasing the potential for an abscopal effect in the future.

Two cases of the abscopal effect on metastatic HNSCC have been described. Shinde and colleagues reported a human papillomavirus-associated oropharyngeal cancer presenting with locoregionally advanced and distantly metastatic ${ }^{10}$. The patient received concurrent ipilimum$\mathrm{ab}$ and nivolumab and showed progression of disease at all sites. Due to increasing symptomatology, the patient underwent palliative radiotherapy ${ }^{25}$. Notably, no radiation was targeted to the lung disease, and both ipilimumab and nivolumab were continued during radiotherapy. Follow-up at 2 weeks post-radiotherapy demonstrated decreased disease burden at all sites, highlighting an abscopal effect.

The abscopal effect was also described by Yazici $e t a l$. in a patient with poorly differentiated carcinoma ${ }^{11}$. Initial treatment included radical locoregional resection followed by adjuvant chemoradiotherapy. The patient had continued locoregional and distant disease progression despite the addition of second-line chemotherapy. Treatment with pembrolizumab offered partial response, but continued disease progression prompted hypofractionated stereotactic radiotherapy with 24 Gy in 3 fractions. Continued immunotherapy and additional hypofractionated stereotactic radiotherapy resulted in a complete response to all lesions, demonstrating a full abscopal effect.

\section{SUMMARY}

The currently presented case is the first case of the abscopal effect in a patient with metastatic HNSCC treated with palliative radiotherapy concurrent with single-agent nivolumab immunotherapy, and only the 3rd documented instance of the abscopal effect in HNSCC to date. In a patient demonstrating oligometastatic progression while treated with nivolumab, the addition of palliative radiotherapy resulted in a dramatic, sustained reduction in disease burden. We also offer the first systematic review of the abscopal effect in metastatic HNSCC and highlight two important cases. The presented case also highlights the potential important role dual treatment with immunotherapy and radiotherapy may play in the treatment of metastatic HNSCC.

\section{CONFLICT OF INTEREST DISCLOSURES}

We have read and understood Current Oncology's policy on disclosing conflicts of interest, and we declare that we have none.

\section{AUTHOR AFFILIATIONS}

*Division of Otolaryngology-Head and Neck Surgery, Department of Surgery, Queen Elizabeth II Health Sciences Centre and Dalhousie University, Halifax, NS; 'Department of OtolaryngologyHead and Neck Surgery, Medical University of South Carolina, Charleston, SC, U.S.A.; ${ }^{\ddagger}$ Department of Radiation Oncology, ${ }^{\circledR}$ Department of Pathology, and ${ }^{\|}$Division of Medical Oncology, Department of Medicine, Queen Elizabeth II Health Sciences Centre and Dalhousie University, Halifax, NS.

\section{REFERENCES}

1. UICC. Locally Advanced Squamous Carcinoma of the Head and Neck; 2014 Review of Cancer Medicines on the WHO List of Essential Medicines. 2014.

2. Ansa B, Goodman M, Ward K, et al. Paranasal sinus squamous cell carcinoma incidence and survival based on Surveillance, Epidemiology, and End Results data, 1973 to 2009. Cancer 2013;119:2602-10.

3. Kazi M, Awan S, Junaid M, Qadeer S, Hassan NH. Management of sinonasal tumors: prognostic factors and outcomes: a 10-year experience at a tertiary care hospital. Indian J Otolaryngol Head Neck Surg 2013;65:155-9.

4. Guntinas-Lichius O, Kreppel M, Stuetzer H, Semrau R, Eckel $\mathrm{H}$, Mueller R. Single modality and multimodality treatment of nasal and paranasal sinuses cancer: a single institution experience of 229 patients. Eur J Surg Oncol 2007;33:222-8.

5. Ferris RL, Blumenschein Jr G, Fayette J, et al. Nivolumab for recurrent squamous-cell carcinoma of the head and neck. N Engl J Med 2016;375:1856-67.

6. Ferris RL, Blumenschein Jr G, Fayette J, et al. Nivolumab vs investigator's choice in recurrent or metastatic squamous cell carcinoma of the head and neck: 2-year long-term survival update of CheckMate 141 with analyses by tumor PD-L1 expression. Oral Oncol 2018;81:45-51.

7. Szturz P, Vermorken JB. Immunotherapy in head and neck cancer: aiming at EXTREME precision. BMC Med 2017;15:110.

8. Burtness B, Harrington K, Greil R, et al. KEYNOTE-048: phase III study of first-line pembrolizumab $(\mathrm{P})$ for recurrent/metastatic head and neck squamous cell carcinoma (R/M HNSCC). Ann Oncol 2018;29(suppl 8):mdy424.045.

9. Grimaldi AM, Simeone E, Giannarelli D, et al. Abscopal effects of radiotherapy on advanced melanoma patients who progressed after ipilimumab immunotherapy. Oncoimmunology 2014;3:e28780.

10. Shinde A, Novak J, Freeman ML, Glaser S, Amini A. Induction of the abscopal effect withimmunotherapy and palliative radiation in metastatic head and neck squamous cell carcinoma: a case report and review of the literature. Cureus 2019;11:e4201.

11. Yazici G, Gullu I, Cengiz M, et al. The synergistic effect of immune checkpoint blockade and radiotherapy in recurrent/ metastatic sinonasal cancer. Cureus 2018;10:e3519.

12. Gagnier JJ, Kienle G, Altman DG, Moher D, Sox H, Riley D. 
The CARE guidelines: consensus-based clinical case reporting guideline development. J Med Case Rep 2013;7:223.

13. Harisiadis L, Kopelson G, Chang CH. Lymphopenia caused by cranial irradiation in children receiving craniospinal radiotherapy. Cancer 1977;40:1102-8.

14. Blomgren H, Glas U, Melén B, Wasserman J. Blood lymphocytes after radiation therapy of mammary carcinoma. Acta Radiol Ther Phys Biol 1974;13:185-200.

15. Cao MD, Chen ZD, Xing Y. Gamma irradiation of human dendritic cells influences proliferation and cytokine profile of T cells in autologous mixed lymphocyte reaction. Cell Biol Int 2004;28:223-8.

16. Vanpouille-Box C, Diamond JM, Pilones KA, et al. TGF $\beta$ is a master regulator of radiation therapy-induced antitumour immunity. Cancer Res 2015;75:2232-42.

17. Kachikwu EL, Iwamoto KS, Liao YP, et al. Radiation enhances regulatory T cell representation. Int J Radiat Oncol Biol Phys 2011;81:1128-35.

18. Liu Y, Dong Y, Kong L, Shi F, Zhu H, Yu J. Abscopal effect of radiotherapy combined with immune checkpoint inhibitors. J Hematol Oncol 2018;11:1-15.
19. Kamperis E, Kodona C, Markou K. Abscopal effect in head and neck cancer: a unicorn summoned once every eon. J Cancer Prev Curr Res 2019;10:115-17.

20. Mole R. Whole body irradiation-radiobiology or medicine? Br J Radiol 1953;26:234-41.

21. Abuodeh Y, Venkat P, Kim S. Systematic review of case reports on the abscopal effect. Curr Prob Cancer 2016;40:25-37.

22. Johnson CB, Jagsi R. The promise of the abscopal effect and the future of trials combining immunotherapy and radiation therapy. Int J Radiat Oncol Biol Phys 2016;95:1254-6.

23. Gunderson AJ, Young KH. Exploring optimal sequencing of radiation and immunotherapy combinations. Adv Radiat Oncol 2018;3:494-505.

24. Dewan MZ, Galloway AE, Kawashima N, et al. Fractionated but not single-dose radiotherapy induces an immunemediated abscopal effect when combined with anti-CTLA-4 antibody. Clin Cancer Res 2009;15:5379-88.

25. Corry J, Peters LJ, D'Costa I, et al. The "QUAD SHOT"- a phase II study of palliative radiotherapy for incurable head and neck cancer. Radiother Oncol 2005;77:137-42. 